\title{
A NOTE ON SIMPLE EIGENVALUES OF MATRIX POLYNOMIALS IN QUEUEING MODELS WITH ERLANG DISTRIBUTIONS
}

\author{
HSING LUH* AND HSIN-YI LIU
}

\begin{abstract}
In this note, we study an $E_{k} / E_{m} / 1$ queueing model where interarrival and service times are Erlang distributions with parameters $k$ and $m$ respectively. We prove that the roots of the characteristic polynomial associated with Erlang distributions are simple if the arrival and service rates are real. Based on this result, a general solution space of vectors for stationary probabilities is easily constructed.
\end{abstract}

AMS Mathematics Subject Classification: 65C40, 60J27.

Key words and phrases: Matrix polynomials, Erlang distribution, quasibirth-and-death process.

\section{Introduction}

We study an $E_{k} / E_{m} / 1$ queueing model where interarrival and service times are Erlang distributions with parameters $k$ and $m$ respectively. Customers are served under the First-come First-served discipline (FCFS). A state of system is denoted by $(n, i, j)$ where $n$ is the number of customers in the service or in the waiting room, $n \geq 0$, and $i$ (resp. $j$ ) is the phase of the customer presenting in the interarrival fictitious center (resp. the service center), $1 \leq i \leq k, 1 \leq$ $j \leq m$. Denote by $F_{T a}(\cdot)$ and $\left(\boldsymbol{\beta}_{1}, \mathbf{S}_{1}\right)$ the interarrival time distribution and its representation of dimension $k$ respectively where the $\boldsymbol{\beta}_{1}=(1,0, \ldots, 0)$ is a $1 \times k$ row vector and $\mathbf{S}_{1}$ is a squared matrix of dimension $k$, i.e., The average arrival rate is simply $\lambda / k$. The distribution function is given by

$$
F_{T a}(t)=1-\boldsymbol{\beta}_{1} \exp \left(\mathbf{S}_{1} t\right) \mathbf{1},
$$

Received August 22, 2005. Revised December 29, 2005. * Corresponding author.

Research partially supported by a grant from National Science Council of Taiwan under No. 92-2219-E-004-004

(C) 2006 Korean Society for Computational \& Applied Mathematics and Korean SIGCAM. 


$$
\mathbf{S}_{1}=\left[\begin{array}{rrrrr}
-\lambda & \lambda & & & \\
& -\lambda & \lambda & & \\
& & \ddots & \ddots & \\
& & & -\lambda & \lambda \\
& & & & -\lambda
\end{array}\right]
$$

where $\mathbf{1}$ is a column vector of all entries equal to 1 . Denote by $f_{T_{a}}^{*}(x)$ the Laplace Stieltjes Transform of $F_{T_{a}}(t)$.

Similarly, the service time distribution $F_{T s}(\cdot)$ has average service rate $\mu / m$ and representation $\left(\boldsymbol{\beta}_{2}, \mathbf{S}_{2}\right)$ of dimension $m$, where $\boldsymbol{\beta}_{2}$ is a $1 \times m$ row vector and $\mathbf{S}_{2}$ is the squared matrix of dimension $m$. The distribution is given by

$$
F_{T s}(t)=1-\boldsymbol{\beta}_{2} \exp \left(\mathbf{S}_{2} t\right) \mathbf{1} .
$$

This model is known as a classic Quasi-Birth-and-Death (QBD) process with phase-type probability distributions. We arrange the states $(n, i, j)$ in lexicographic order, i.e., $(0,1), \ldots,(0, k),(1,1,1), \ldots,(1,1, m),(1,2,1), \ldots,(1,2, m)$, .... Then the transition rate matrix (or infinitesimal generator) $\mathbf{Q}$ is of the block-tridiagonal form and can be written as

$$
\mathbf{Q}=\left[\begin{array}{ccccc}
\mathbf{B}_{0} & \overline{\mathbf{A}}_{0} & & & \\
\mathbf{C}_{0} & \mathbf{A}_{1} & \mathbf{A}_{0} & & \\
& \mathbf{A}_{2} & \mathbf{A}_{1} & \mathbf{A}_{0} & \\
& & \mathbf{A}_{2} & \mathbf{A}_{1} & \ddots \\
& & & \mathbf{A}_{2} & \ddots \\
& & & & \ddots
\end{array}\right]
$$

The submatrices can be written as Kronecker products and Kronecker sums which were defined by Bellman [2] and denoted by $\otimes$ and $\oplus$ respectively. They are expressed by

$$
\begin{array}{ll}
\mathbf{B}_{0}=\mathbf{S}_{1}, & \mathbf{A}_{0}=\boldsymbol{\gamma}_{1} \boldsymbol{\beta}_{1} \otimes \mathbf{I}_{2} \\
\mathbf{C}_{0}=\mathbf{I}_{1} \otimes \boldsymbol{\gamma}_{2}, & \mathbf{A}_{1}=\mathbf{S}_{1} \oplus \mathbf{S}_{2} \\
\overline{\mathbf{A}}_{0}=\boldsymbol{\gamma}_{1} \boldsymbol{\beta}_{1} \otimes \boldsymbol{\beta}_{2}, & \mathbf{A}_{2}=\mathbf{I}_{1} \otimes \boldsymbol{\gamma}_{2} \boldsymbol{\beta}_{2} \\
\boldsymbol{\gamma}_{1}=-\mathbf{S}_{1} \mathbf{1}, & \boldsymbol{\gamma}_{2}=-\mathbf{S}_{2} \mathbf{1},
\end{array}
$$

where $\mathbf{A}_{i}, i=0,1,2$ are squared matrices of dimension $m k, \mathbf{B}_{0}$ is a squared matrix of dimension $k, \mathbf{C}_{0}$ a matrix of dimension $m k$ by $k$, and $\overline{\mathbf{A}}_{0}$ a $k$ by $m k$ matrix.

Suppose the stationary probability of system state is denoted by $\boldsymbol{\pi}$ as

$$
\boldsymbol{\pi}=\left(\boldsymbol{\pi}_{0}, \boldsymbol{\pi}_{1}, \boldsymbol{\pi}_{2}, \ldots\right),
$$

where $\boldsymbol{\pi}_{n}$ is a row vector of stationary probability when there are $n$ customers in the system. To assure a stable system we assume $\lambda / \mu<k / m$ implying the 
existence of $\boldsymbol{\pi}$. For solving $\boldsymbol{\pi}$ which satisfies the state balance equations $\boldsymbol{\pi} \mathbf{Q}=\mathbf{0}$ and the normalization condition $\boldsymbol{\pi} \mathbf{1}=1$, it gives the following equations:

$$
\begin{aligned}
\boldsymbol{\pi}_{0} \mathbf{B}_{0}+\boldsymbol{\pi}_{1} \mathbf{C}_{0} & =\mathbf{0} \\
\boldsymbol{\pi}_{0} \overline{\mathbf{A}}_{0}+\boldsymbol{\pi}_{1} \mathbf{A}_{1}+\boldsymbol{\pi}_{2} \mathbf{A}_{2} & =\mathbf{0} \\
\boldsymbol{\pi}_{n-1} \mathbf{A}_{0}+\boldsymbol{\pi}_{n} \mathbf{A}_{1}+\boldsymbol{\pi}_{n+1} \mathbf{A}_{2} & =\mathbf{0}, \quad n \geq 2, \\
\boldsymbol{\pi} \mathbf{1} & =1 .
\end{aligned}
$$

There are a number of methods to find the stationary probabilities of recurrent QBD processes. Matrix analytic methods have been proved useful in the study of Markov chains of phase-type distributions, providing a solution tool which is relevant for queueing theory and computing probability distributions in many applications. The general approach was introduced by Neuts in [11] where the matrix-geometric method relies on determining the minimal nonnegative matrix solution $R$ of a matrix-quadratic equation; some stationary probability vectors are expressed in terms of powers of $R$. Recently, instead of solving $R$, one solves (3) as a homogeneous difference equation in which the coefficients are no more scalars. To use this method, one must compute the spectral expansion of the stationary state distribution of the system. This representation requires explicit knowledge of eigenvalues and eigenvectors. It requires finding all singularities of a given matrix function in the unit disk and then plugging them into (4) to obtain a set of linear equations in a finite number of unknown boundary probabilities in (1). The remaining probabilities and other measures of interest are then computed from the boundary probabilities. Based on this approach, unboundary state probabilities can be written as a linear combination of vector product-forms, thereupon considerably reducing the computational complexity of solving a complicated problem in QBD processes.

A fundamental idea of this approach is to substitute independent identical distributions by conditional independence and conditional distributions given a QBD process. The efficiency of the method is derived from an algebraic theory which gives the exact decomposition of the eigenvalue problem into small coupled problems and expresses the eigenvectors in Kronector product form. Thus, when the arrivals and servers are identical or can be grouped into classes, the eigenvalues are given as roots of polynomials of small degree and the eigenvectors are given in closed form. A number of authors have given different names for this method. Liefvoort [8] introduced a coupling matrix whose eigenvalues are solutions to the functional equation

$$
f_{T a}^{*}(x) f_{T s}^{*}(-x)=1 .
$$

Bertsimas [3] used (5) which is deduced in $C_{k} / C_{m} / s$ where both interarrival times and service times have phase type probability distributions and proved that the stationary probability of $n$ customers in system is a linear combination of geometric terms when $n \geq s$. Le Boudec [4] studied a $P H / P H / 1$ queue. He 
showed that the stationary solution is a linear combination of Kronecker productforms which can be expressed in terms of roots of the associated characteristic polynomial. Luh [9] used a similar approach to derive stationary probabilities in terms of linear combinations of product-forms in studying a system of two stations in tandem. In short, we concentrate on what will be called the Kronecker vector approach. These results are useful in deriving bounds as well as approximations of tail probabilities. Particularly, it will greatly reduce the computation time.

In general, (5) may induce multiple roots. However, there was no theoretic discussion of such a case in $[1,3,4,9]$. They all presented that the transforms of interarrival and service times distributions satisfy an equation of a simple form, and used the roots of the equation to express the stationary solution under the assumption all the roots are distinct. Lately, Grassmann adopted (5) to find zeros in order to study two specified queueing models [5,6]. Again, it was assumed that there is no multiple root in (5). In order to investigate the property of (5), we study a specific queueing model of QBD process in this note. We prove that for any positive integer $k$ and $m$, except zero there are only simple roots in (5) derived from $E_{k} / E_{m} / 1$. For the case of multiple roots arising in general queueing models, readers may refer to [10].

\section{Singularities of $\mathbf{Q}(w)$ in the open unit disk}

We consider the equations of the stationary states for $n \geq 1$ and study the special solution in (3) of the homogeneous difference equations. We assume the associated stationary probabilities are of the form

$$
\varphi_{n}=w^{n} \mathbf{y}, n \geq 1
$$

where $w \neq 0$ is a complex number and $\mathbf{y}$ is a $1 \times m k$ vector. Initially, set $\boldsymbol{\pi}_{n}=\boldsymbol{\varphi}_{n}$. From (3), we have for $n \geq 1$

$$
w^{n} \mathbf{y}\left(\mathbf{A}_{0}+w \mathbf{A}_{1}+w^{2} \mathbf{A}_{2}\right)=\mathbf{0} .
$$

Define a fundamental matrix polynomial

$$
\mathbf{Q}(w) \triangleq \mathbf{A}_{0}+w \mathbf{A}_{1}+w^{2} \mathbf{A}_{2} .
$$

If $w_{o}$ satisfies $\operatorname{det} \mathbf{Q}\left(w_{o}\right)=0$, then $w_{o}$ is called a singularity of $\mathbf{Q}(w)$.

Denote by $w_{i}$ and $\mathbf{y}_{i}$ the singularities and corresponding left vectors of $\mathbf{Q}(w)$, respectively. It implies

$$
\mathbf{y}_{i} \mathbf{Q}\left(w_{i}\right)=\mathbf{0}, \text { for } i=1,2, \ldots, d,
$$

where $d$ is the degree of $\operatorname{det} \mathbf{Q}(w)$. At the study in [8], it has been proved that $d$ is a finite number. Clearly, for every $w_{i}$ and a corresponding vector $\mathbf{y}_{i}$, the sequence

satisfies (6).

$$
\left\{w_{i}^{n} \mathbf{y}_{i}: n \geq 1\right\}
$$


In what follows, we look for the nonzero roots of $\operatorname{det} \mathbf{Q}(w)=0$ and the corresponding vectors. Replace $\mathbf{A}_{0}, \mathbf{A}_{1}, \mathbf{A}_{2}$ in (6) by $\boldsymbol{\gamma}_{1} \boldsymbol{\beta}_{1} \otimes \mathbf{I}_{2}, \mathbf{S}_{1} \oplus \mathbf{S}_{2}$ and $\mathbf{I}_{1} \otimes \boldsymbol{\gamma}_{2} \boldsymbol{\beta}_{2}$, then (6) becomes

$$
w^{n-1} \mathbf{y}\left[\left(\boldsymbol{\gamma}_{1} \boldsymbol{\beta}_{1} \otimes \mathbf{I}_{2}\right)+w\left(\mathbf{S}_{1} \oplus \mathbf{S}_{2}\right)+w^{2}\left(\mathbf{I}_{1} \otimes \boldsymbol{\gamma}_{2} \boldsymbol{\beta}_{2}\right)\right]=\mathbf{0},
$$

which is equivalent to

$$
\mathbf{y}\left[\left(w \mathbf{S}_{1}+\boldsymbol{\gamma}_{1} \boldsymbol{\beta}_{1}\right) \oplus\left(w \mathbf{S}_{2}+w^{2} \boldsymbol{\gamma}_{2} \boldsymbol{\beta}_{2}\right)\right]=\mathbf{0},
$$

since $\frac{1}{w}\left(\boldsymbol{\gamma}_{1} \boldsymbol{\beta}_{1} \otimes \mathbf{I}_{2}\right)=\left(\frac{1}{w} \boldsymbol{\gamma}_{1} \boldsymbol{\beta}_{1} \otimes \mathbf{I}_{2}\right)$ and $w\left(\mathbf{I}_{1} \otimes \boldsymbol{\gamma}_{2} \boldsymbol{\beta}_{2}\right)=\left(\mathbf{I}_{1} \otimes w \boldsymbol{\gamma}_{2} \boldsymbol{\beta}_{2}\right)$. Our goal is to find nonzero $w$ satisfying the condition in which the determinant of $\mathbf{Q}(w)$ is zero. Define

$$
\sigma(A)=\text { the set of eigenvalues of } A \text {. }
$$

Theorem 1. If $\epsilon \in \sigma\left(w \mathbf{S}_{1}+\boldsymbol{\gamma}_{1} \boldsymbol{\beta}_{1}\right)$ is an eigenvalue whose corresponding eigenvector is $\mathbf{u} \in \mathcal{C}^{k}$ and if $\xi \in \sigma\left(w \mathbf{S}_{2}+w^{2} \boldsymbol{\gamma}_{2} \boldsymbol{\beta}_{2}\right)$ is also an eigenvalue whose corresponding eigenvector is $\mathbf{v} \in \mathcal{C}^{m}$, then $\epsilon+\xi$ is an eigenvalue of the Kronecker sum, $\left(w \mathbf{S}_{1}+\boldsymbol{\gamma}_{1} \boldsymbol{\beta}_{1}\right) \oplus\left(w \mathbf{S}_{2}+w^{2} \boldsymbol{\gamma}_{2} \boldsymbol{\beta}_{2}\right)$, and $\mathbf{u} \otimes \mathbf{v} \in \mathcal{C}^{k m}$ is a corresponding eigenvector. If $\sigma\left(w \mathbf{S}_{1}+\boldsymbol{\gamma}_{1} \boldsymbol{\beta}_{1}\right)=\left\{\epsilon_{1}, \cdots, \epsilon_{k}\right\}$ and $\sigma\left(w \mathbf{S}_{2}+w^{2} \boldsymbol{\gamma}_{2} \boldsymbol{\beta}_{2}\right)=\left\{\xi_{1}, \cdots, \xi_{m}\right\}$, then $\sigma\left(\left(w \mathbf{S}_{1}+\boldsymbol{\gamma}_{1} \boldsymbol{\beta}_{1}\right) \oplus\left(w \mathbf{S}_{2}+w^{2} \boldsymbol{\gamma}_{2} \boldsymbol{\beta}_{2}\right)\right)=\left\{\epsilon_{i}+\xi_{j} \mid i=1,2, \cdots, k, j=1, \cdots, m\right\}$.

The proof is given in Theorem 4.4.5 in section 4.4 of [7]. Define

$$
\begin{aligned}
& \mathbf{a}(w) \triangleq w \mathbf{S}_{1}+\boldsymbol{\gamma}_{1} \boldsymbol{\beta}_{1}, \\
& \mathbf{b}(w) \triangleq w \mathbf{S}_{2}+w^{2} \boldsymbol{\gamma}_{2} \boldsymbol{\beta}_{2} .
\end{aligned}
$$

Thus, it is easy to prove $\mathbf{Q}(w)=\mathbf{a}(w) \oplus \mathbf{b}(w)$.

Given $w$, there is a close connection between the singularity of $\mathbf{Q}(w)$ and an equation involving the Laplace transforms which is defined by (5). Since the eigenvalue of Kronecker sum of $\mathbf{a}(w) \oplus \mathbf{b}(w)$ is the sum of the eigenvalues of $\mathbf{a}(w)$ and $\mathbf{b}(w)$, we shall prove $\operatorname{det} \mathbf{Q}(w)=0$ if and only if there exists $x$ such that $\operatorname{det}\left(\mathbf{a}-x \mathbf{I}_{1}\right)=0$ and $\operatorname{det}\left(\mathbf{b}+x \mathbf{I}_{2}\right)=0$. In other words, given $w \neq 0$, $\operatorname{det}(\mathbf{a}(w) \oplus \mathbf{b}(w))=0$ if and only if $w=f_{T a}^{*}(x)$ where $x$ satisfies (5). It is because

$$
\operatorname{det}(\mathbf{a}(w) \oplus \mathbf{b}(w))=w^{m k} \operatorname{det}\left(\frac{1}{w} \mathbf{a}(w) \oplus \frac{1}{w} \mathbf{b}(w)\right)=0
$$

if and only if there exists $x$ such that

$$
\left\{\begin{array}{r}
\operatorname{det}\left(\frac{1}{w} \mathbf{a}(w)-x \mathbf{I}_{1}\right)=0 \\
\operatorname{det}\left(\frac{1}{w} \mathbf{b}(w)+x \mathbf{I}_{2}\right)=0 .
\end{array}\right.
$$

Lemma 1. Let $w \neq 0$. We shall show

$$
\begin{aligned}
& \text { (1) } \operatorname{det}\left(\frac{1}{w} \mathbf{a}(w)-x \mathbf{I}_{1}\right)=\left(1-\frac{1}{w} f_{T a}^{*}(x)\right)(-\lambda-x)^{k}, \\
& \text { (2) } \operatorname{det}\left(\frac{1}{w} \mathbf{b}(w)+x \mathbf{I}_{2}\right)=\left(1-w f_{T s}^{*}(-x)\right)(x-\mu)^{m} .
\end{aligned}
$$


Proof. (1) Compute $\operatorname{det}\left(\frac{1}{w} \mathbf{a}(w)-x \mathbf{I}_{1}\right)$ and it gives

$$
\begin{aligned}
\operatorname{det}\left(\frac{1}{w} \gamma_{1} \boldsymbol{\beta}_{1}+\mathbf{S}_{1}-x \mathbf{I}_{1}\right) & =\left[\begin{array}{ccccc}
-\lambda-x & \lambda & 0 & \cdots & 0 \\
& -\lambda-x & \lambda & \cdots & 0 \\
\vdots & \vdots & \ddots & \ddots & \vdots \\
\frac{1}{w} \lambda & 0 & 0 & -\lambda-x & \lambda \\
& 0 & 0 & 0 & -\lambda-x
\end{array}\right] \\
& =(-\lambda-x)^{k}+(-1)^{k+1} \frac{\lambda^{k}}{w} \\
& =(-\lambda-x)^{k}\left[1+(-1)^{k+1} \frac{1}{w} \frac{\lambda^{k}}{(-\lambda-x)^{k}}\right]
\end{aligned}
$$

A similar argument is applied to prove (2). It may be skipped here.

Lemma 2. Let $w \neq 0, \operatorname{det}(\mathbf{a}(w) \oplus \mathbf{b}(w))=0$ if and only if $w=f_{T a}^{*}(x)$ where $x$ satisfies (5).

Proof. For $x \neq-\lambda_{i}, \mu_{i}$ for all $i$, then (7) holds if and only if $w=f_{T a}^{*}(x)$ where $x$ satisfies (5) by Lemma 1 . To show it holds for $x=-\lambda_{i}$ or $\mu_{i}$, one needs to consider $\mathbf{S}_{1}-\varepsilon \mathbf{I}_{1}$ or $\mathbf{S}_{2}+\varepsilon \mathbf{I}_{2}$ in place of $\mathbf{S}_{1}$ and $\mathbf{S}_{2}$ with a small constant $\varepsilon$, and by continuity argument, the lemma is proved.

\section{Simple roots in $E_{k} / E_{m} / 1$}

In this section, we show that in $E_{k} / E_{m} / 1$ system nonzero roots of (5) are simple. Without loss generality, we assume that the arrival rate $\lambda$ and service rate $\mu$ are positive real numbers. The following theorem holds true.

Theorem 2. For any $\lambda>0$ and $\mu>0$, the nonzero roots of (5), i.e.,

$$
\left(\frac{\lambda}{x+\lambda}\right)^{k}\left(\frac{\mu}{\mu-x}\right)^{m}=1
$$

are simple.

Before proving this result, we first recall the Jensen's inequality theorem.

Theorem 3. (Jensen's inequality) Let $\phi$ be convex over real line $(a, b)$. Let $\left\{x_{j}\right\}_{j=1}^{N}$ be points of $(a, b)$ and $\left\{p_{j}\right\}_{j=1}^{N}$ satisfy $p_{j} \geq 0$ and $\sum p_{j}>0$. Then we have

$$
\phi\left(\frac{\sum p_{j} x_{j}}{\sum p_{j}}\right) \leq \frac{\sum p_{j} \phi\left(x_{j}\right)}{\sum p_{j}} .
$$


Proof of Theorem 2. It is sufficient to consider the function

$$
g(x) \triangleq \lambda^{k} \mu^{m}-(x+\lambda)^{k}(\mu-x)^{m} .
$$

Since

$$
g^{\prime}(x)=(\lambda+x)^{k-1}(\mu-x)^{m-1}[(m+k) x-(k \mu-m \lambda)],
$$

it follows that the possible multiple roots only occur at $x=-\lambda$, $\mu$, and $\frac{k \mu-m \lambda}{m+k}$. Obviously, $g(-\lambda) \neq 0$ and $g(\mu) \neq 0$. We only need to consider $\frac{k \mu-m \lambda}{m+k}$ and denote it by $x_{0}$.

Let

$$
t \triangleq \frac{m}{k}
$$

Then $t$ is a positive real number and

$$
g\left(x_{0}\right)=\left(\lambda \mu^{t}\right)^{k}-\left[\left(\frac{\lambda+\mu}{1+t}\right)^{1+t} t^{t}\right]^{k} .
$$

Since $\lambda, \mu$, and $t$ are real, $g\left(x_{0}\right)=0$ leads to

$$
\lambda \mu^{t}=\left(\frac{\lambda+\mu}{1+t}\right)^{1+t} t^{t}
$$

That is, $g\left(x_{0}\right)=0$ if and only if $\lambda, \mu$, and $t$ satisfy the equation

$$
\lambda\left(\frac{\mu}{t}\right)^{t}=\left(\frac{\lambda+\mu}{1+t}\right)^{1+t}
$$

In the following argument, we use the Jensen's inequality to verify that (8) holds for any properly selected positive real numbers $\lambda, \mu$, and $t$.

Given any $t>0$, let

$$
\phi(x)=e^{(t+1) x}
$$

Because of

$$
\phi^{\prime}(x)=(t+1) e^{(t+1) x}>0
$$

and

$$
\phi^{\prime \prime}(x)=(t+1)^{2} e^{(t+1) x}>0,
$$

it follows that $\phi(x)$ is convex over $(-\infty, \infty)$. Let $x_{1}=\ln \lambda^{\frac{1}{t+1}}, x_{2}=\ln \left(\frac{\mu}{t}\right)^{\frac{1}{t+1}}$, $p_{1}=\frac{1}{t+1}$, and $p_{2}=\frac{t}{t+1}$. Jensen's inequality theorem implies

$$
\phi\left(\frac{p_{1} x_{1}+p_{2} x_{2}}{p_{1}+p_{2}}\right) \leq \frac{p_{1} \phi\left(x_{1}\right)+p_{2} \phi\left(x_{2}\right)}{p_{1}+p_{2}} .
$$




$$
\begin{aligned}
& \text { Left of (9) }=\phi\left(\frac{1}{t+1} \ln \lambda^{\frac{1}{t+1}}+\frac{t}{t+1} \ln \left(\frac{\mu}{t}\right)^{\frac{1}{t+1}}\right) \\
& =e^{\ln \lambda^{\frac{1}{t+1}}+t \ln \left(\frac{\mu}{t}\right)^{\frac{1}{t+1}}} \\
& =e^{\ln \lambda^{\frac{1}{t+1}}} \cdot e^{\ln \left(\frac{\mu}{t}\right)^{\frac{t}{t+1}}} \\
& =\left[\lambda\left(\frac{\mu}{t}\right)^{t}\right]^{\frac{1}{t+1}} \text {. } \\
& \text { Right of }(9)=\frac{1}{t+1} e^{(1+t) \ln \lambda^{\frac{1}{t+1}}}+\frac{t}{t+1} e^{(t+1) \ln \left(\frac{\mu}{t}\right)^{\frac{1}{t+1}}} \\
& =\frac{1}{t+1} e^{\ln \lambda}+\frac{t}{t+1} e^{\ln \left(\frac{\mu}{t}\right)} \\
& =\frac{1}{t+1}\left[\lambda+t\left(\frac{\mu}{t}\right)\right] \\
& =\frac{1}{t+1}(\lambda+\mu) \text {. }
\end{aligned}
$$

We obtain

$$
\lambda\left(\frac{\mu}{t}\right)^{t} \leq\left(\frac{\lambda+\mu}{t+1}\right)^{t+1} .
$$

Since $\phi(x)$ is convex, the equality holds in (9) if and only if $x_{1}=x_{2}$. Hence, it yields

$$
\lambda\left(\frac{\mu}{t}\right)^{t}=\left(\frac{\lambda+\mu}{t+1}\right)^{t+1}
$$

if and only if $\frac{\mu}{t}=\lambda$, i.e., $x_{0}=0$.

It implies if there exists any multiple root of (5), it must be $x=0$. Hence, any other nonzero root of (5) is simple.

Theorem 4. In $E_{k} / E_{m} / 1$, there are no multiple roots except zero of (5). Assume there are $d$ nonzero roots of it. Let $w_{1}, \ldots, w_{d}$ be distinct singularities of $\mathbf{Q}(w)$ in the open unit disk where $\mathbf{y}_{i}$ are the corresponding eigenvectors. Given $w_{i}$, let $\boldsymbol{\varphi}_{n}\left(w_{i}\right)=w_{i}^{n} \mathbf{y}_{i}, n \geq 1$. Then the stationary probability $\boldsymbol{\pi}_{n}$ is easily constructed by

$$
\boldsymbol{\pi}_{n}=\sum_{i=1}^{d} c_{i} \boldsymbol{\varphi}_{n}\left(w_{i}\right), \quad \text { for } n \geq 2
$$

where $c_{i}$ are determined such that the boundary equation (1) and the normalized condition (4) are satisfied.

\section{Illustrative examples}

To describe the theorems presented before, we give numerical examples for showing the solution procedure in this section. 


\subsection{An example of $M / M / 1$ system}

The system has the following features:

$$
\lambda=4, \quad \mu=5 .
$$

Step 1. Solve equation $(5), f_{T_{a}}^{*}(x) f_{T_{s}}^{*}(-x)=1$. Let $x$ be a solution with the positive real part of (5). Because

$$
f_{T_{a}}^{*}(x)=\left(\frac{4}{x+4}\right), \quad f_{T_{s}}^{*}(x)=\left(\frac{5}{x+5}\right),
$$

the solutions of $f_{T_{a}}^{*}(x) f_{T_{s}}^{*}(-x)=1$ is

$$
x_{1}=1.0000 \text {. }
$$

Step 2. For solutions $w, \mathbf{u}, \mathbf{v}$ defined in Theorem 1. Obtain $w=0.8000, \mathbf{u}=$ 1.0000 , and $\mathbf{v}=1.0000$.

Step 3. Compute $\boldsymbol{\varphi}_{n}(w)$ defined in Theorem 4, i.e., $\boldsymbol{\varphi}_{n}(w)=w^{n}(\mathbf{u} \otimes \mathbf{v}), n \geq 1$.

$$
\begin{aligned}
& \boldsymbol{\varphi}_{1}(w)=0.8000, \\
& \boldsymbol{\varphi}_{2}(w)=0.6400, \\
& \boldsymbol{\varphi}_{3}(w)=0.5120, \\
& \boldsymbol{\varphi}_{4}(w)=0.4096, \\
& \boldsymbol{\varphi}_{5}(w)=0.3277, \\
& \boldsymbol{\varphi}_{6}(w)=0.2621 .
\end{aligned}
$$

Step 4. Let $\boldsymbol{\pi}_{n}$ be a linear combination of $\boldsymbol{\varphi}_{n}(w)$ that is $\boldsymbol{\pi}_{n}=c \boldsymbol{\varphi}_{n}(w), c \in \mathbb{C}$.

Step 5. Set a linear nonhomogeneous system consisting of equations (1), (2), and (4).

Step 6. Obtain coefficient $c$ and $\boldsymbol{\pi}_{0}$.

$$
\begin{gathered}
\pi_{0}=0.2000, \\
c=0.2000 .
\end{gathered}
$$

Step 7. Substitute coefficients $c$ and obtain stationary probabilities $\boldsymbol{\pi}_{n}$ by Theorem 4 .

$$
\begin{aligned}
& \pi_{1}=0.1600, \\
& \pi_{2}=0.1280, \\
& \pi_{3}=0.1024, \\
& \pi_{4}=0.4096, \\
& \pi_{5}=0.3277, \\
& \pi_{6}=0.2621 .
\end{aligned}
$$




\subsection{An example of $E_{2} / E_{2} / 1$ system}

The second example pertains to the $E_{2} / E_{2} / 1$ System. The system has the following features:

$$
\boldsymbol{\beta}_{1}=\boldsymbol{\beta}_{2}=(1,0), \quad \lambda_{1}=\lambda_{2}=4, \quad \mu_{1}=\mu_{2}=5 .
$$

Step 1. Let $x_{i}$ be a solution with positive real parts of (5), $i=1,2$. Because

$$
f_{T_{a}}^{*}(x)=\left(\frac{4}{x+4}\right)^{2}, \quad f_{T_{s}}^{*}(x)=\left(\frac{5}{x+5}\right)^{2},
$$

the solutions of $f_{T_{a}}^{*}(x) f_{T_{s}}^{*}(-x)=1$ are

$$
x_{1}=1.0000, \quad x_{2}=6.8443 .
$$

Step 2. Compute

(1)

$$
\begin{gathered}
w_{1}=f_{T_{a}}^{*}\left(x_{1}\right)=0.64, \\
w_{2}=f_{T_{a}}^{*}\left(x_{2}\right)=0.1361 .
\end{gathered}
$$

(2) by $\mathbf{u}_{i}=\mathbf{e}_{1}\left(\mathbf{S}_{1}-x_{i} \mathbf{I}_{1}\right) \boldsymbol{\beta}_{1}^{\prime} \boldsymbol{\beta}_{1}\left(\mathbf{S}_{1}-x_{i} \mathbf{I}_{1}\right)^{-1}$.

$$
\begin{aligned}
& \mathbf{u}_{\mathbf{1}}=(1.0000,0.8000), \\
& \mathbf{u}_{2}=(1.0000,0.3689) .
\end{aligned}
$$

(3) by $\mathbf{v}_{i}=\mathbf{e}_{2}\left(\mathbf{S}_{2}+x_{i} \mathbf{I}_{2}\right) \boldsymbol{\beta}_{2}^{\prime} \boldsymbol{\beta}_{2}\left(\mathbf{S}_{2}+x_{i} \mathbf{I}_{2}\right)^{-1}$.

$$
\begin{gathered}
\mathbf{v}_{1}=(1.0000,1.2500), \\
\mathbf{v}_{2}=(1.0000,-2,7111) .
\end{gathered}
$$

Step 3. Compute $\boldsymbol{\varphi}_{n}\left(w_{i}\right)=w_{i}^{n}\left(\mathbf{u}_{i} \otimes \mathbf{v}_{i}\right)$.

$$
\begin{gathered}
\boldsymbol{\varphi}_{1}\left(w_{1}\right)=(0.6400,0.8000,0.5120,0.6400), \\
\boldsymbol{\varphi}_{2}\left(w_{1}\right)=(0.4096,0.5120,0.3277,0.4096), \\
\boldsymbol{\varphi}_{3}\left(w_{1}\right)=(0.2621,0.3277,0.2097,0.2621), \\
\boldsymbol{\varphi}_{1}\left(w_{2}\right)=(0.1361,-0.3689,0.0502,-0.1361), \\
\boldsymbol{\varphi}_{2}\left(w_{2}\right)=(0.0185,-0.0502,0.0068,-0.0185), \\
\boldsymbol{\varphi}_{3}\left(w_{2}\right)=(0.0025,-0.0068,0.0009,-0.0025),
\end{gathered}
$$

Step 4. Let $\boldsymbol{\pi}_{n}$ be a linear combination of $\boldsymbol{\varphi}_{n}\left(w_{i}\right)$ that is $\boldsymbol{\pi}_{n}=\sum_{i=1}^{2} c_{i} \boldsymbol{\varphi}_{n}\left(w_{i}\right)$, $c_{i} \in \mathbb{C}$.

Step 5. Set a linear nonhomogeneous system consisting of equations (1), (2), and (4). 
Step 6. Obtain coefficients $c_{i}, i=1,2$, and stationary probabilities $\boldsymbol{\pi}_{0}$.

$$
\begin{gathered}
\boldsymbol{\pi}_{0}=(0.0631,0.1369), \\
c_{1}=0.1171, \quad c_{2}=0.1171 .
\end{gathered}
$$

Step 7. Substituting coefficients $c_{i}, i=1,2$, and obtain $\boldsymbol{\pi}_{n}$.

$$
\begin{aligned}
& \boldsymbol{\pi}_{1}=(0.0909,0.0505,0.0658,0.0590), \\
& \boldsymbol{\pi}_{2}=(0.0501,0.0541,0.0392,0.0458), \\
& \boldsymbol{\pi}_{3}=(0.0310,0.0376,0.0247,0.0304) .
\end{aligned}
$$

\section{REFERENCES}

1. Ivo Adan and Yiqiang Zhao, Analyzing GI/Er/1 queues, Operations Research Letters 19(4) (1996), 183-190.

2. R. Bellman, Introduction to Matrix Analysis, MacGraw-Hill, London, 1960.

3. D. Bertsimas, An analytic approach to a general class of $G / G / s$ queueing systems, Operations Research 38 (1990), 139-155.

4. J. Y. Le Boudec, Steady-state probabilities of the PH/PH/1 queue, Queueing Systems 3 (1988), 73-88.

5. W. K. Grassmann, Real eigenvalues of certain tridiagonal matrix polynomials with queueing applications, Linear Algebra and Its Applications 342 (2002), 93-106.

6. W. K. Grassmann and S. Drekic, An analytical solution for a tandem queue with blocking, Queueing Systems 36 (2000), 221-235.

7. R. A. Horn, and C. R. Johnson, Matrix Topics in Marrix, 1991.

8. A. Van De Liefvoort, The waiting-time distribution and its moments of the $P H / P H / 1$ queue, Operations Research Letters 9 (1990), 261-269.

9. H. Luh, Matrix product-form solutions of stationary probabilities in tandem queues, Journal of the Operations Research 42(4) (1999), 436-656.

10. H. Luh and H. Y. Liu, Vector product-form solutions of stationary probability in a singleserver queue with matrix polynomial approaches, in print, 2004.

11. M. F. Neuts, Matrix-Geometric Solutions in Stochastic Models, The John Hopkins University Press, 1981.

Hsing Luh received his Ph. D. in Operations Research at North Carolina State University, USA, 1992. He is professor of Department of Mathematical Sciences, National Chengchi University and president of Operations Research Society of Taiwan. His research interests include Queueing theory and applications, Stochastic models and simulations, Linear programming and optimization. His research accomplishment has been recognized by the international research award and the excellent research lecture award of National Chengchi University.

Department of Mathematical Sciences, National Chengchi University, Taipei 116, Taiwan ROC

e-mail: slu@nccu.edu.tw

Hsin-Yi Liu was a Master student at the Department of Mathematical Sciences, National Chengchi University. She is a high school teacher since finishing her master degree. 